\title{
Biologie et physiologie du syndrome de détresse respiratoire de l'adulte
}

A la suite d'une agression, par exemple endotoxinique, les cellules endothéliales pulmonaires libèrent des substances chimiotactiques et expriment à leur membrane des molécules d'adhérence permettant la fixation de polynucléaires neutrophiles. Les neutrophiles et les macrophages alvéolaires sont sensibilisés, puis activés ; ils relarguent alors toute une série de cytokines et de médiateurs de l'inflammation et attaquent la paroi alvéolaire par l'intermédiaire de protéases, de dérivés toxiques de l'oxygène et de microthrombi vasculaires. Survient ensuite une phase de "réparation " et de fibrose qui altère les propriétés mécaniques du poumon, sa vascularisation et la capacité de diffusion de l'oxygène. Enfin, peut parfois se produire une restitution ad integrum comportant non seulement l'arrêt des processus inflammatoire et de destruction tissulaire, mais aussi une régression de la fibrose.

\section{Alain F. Junod}

\section{ADRESSE}

A.F. Junod: professeur de médecine. Division dc pncumologic, hôpital cantonal universitairc de Genčvc, 24, ruc Michcli-du-Crest, a compréhension des dérèglements fonctionnels du poumon, qu'il s'agisse de pathologie obstructive ou d'affections interstitielles, a atteint maintenant un niveau élevé. Elle est à l'origine d'un certain nombre de progrès dans le traitement de maladies pulmonaires, surtout de celles dont le caractère aigu ou grave requiert l'admission dans une unité de soins intensifs et l'utilisation de mesures de soutien, telles la ventilation mécanique ou même l'oxygénation extracorporelle. L'élucidation des mécanismes cellulaires et biologiques à l'origine de ces troubles de la fonction n'est certainement pas à un stade aussi avancé, même si un effort soutenu a été accompli dans ce domaine, ainsi qu'en atteste le monu- mental ouvrage récemment publić : The Lung : Scientific Foundations [1]. Le présent essai n'a pas la prétention de se substituer aux 2204 pages de ce traité, mais bien plutôt d'essayer de présenter le déroulement de phénomènes à la fois physiologiques et biologiques menant à un état pathologique particulier: le syndrome de détresse respiratoire de l'adulte, afin d'illustrer le changement intervenu dans l'approche pathogénique de ce syndrome.

\section{Structure alvéolocapillaire} (figure 1)

Seule cette région sera brièvement décrite, puisque c'est elle qui est impliquée dans les échanges gazcux. D'un côté, il y a les espaces aériens 
ou alvéolaircs, d'une surface totale équivalant à un court de tennis. Ils sont bordés d'un épithélium fait de deux types cellulaires: lc pncumocyte I, cellule différenciée, incapable de se diviser, couvrant la plus grande partie de l'alvéole, et le pncumocyte II, ccllule chargée de la production du surfactant (substance tensio-active, stabilisant les alvéoles en diminuant la tension de surface rćsultant de l'interface air-liquide), capable de se diviser et de se différencier ultérieurement en pneumocyte I. De l'autre côté, il y a les espaces capillaircs vasculaircs bordés d'un endothélium. Les jonctions interccllulaires, aussi bicn au nivcau endothélial qu'épithélial, sont du type serré, la perméabilité paracellulaire étant la plus faible au niveau de l'épithélium alvéolaire. Entre ces espaces aérien et vasculaire se trouve l'espace interstitiel, fait d'une matrice extracellulairc et de fibroblastes. La matrice extracellulaire elle-même comprend des protéines de structure, telles les fibres collagènes et élastiques, la membrane basale (qui borde les vaisseaux et alvéoles), des glycoprotéines, telles la laminine et la fibronectine (clle-même dotće de plusieurs proprićtés biologiques : promotion de l'adhćrence cellulaire, chémoattraction, stimulation de la prolifération), ainsi que des protéoglycanes. Les macrophages alvéolaires résident dans les espaces alvéolaires. Ils dérivent des monocytes circulants et représentent un état de différenciation ultime. Ils font partic des mécanismes de défensc pulmonaire, étant capables de phagocyter et tuer bon nombre de micro-organismes, de produire des radicaux libres de l'oxygène et des protéases, et de libérer plusicurs cytokines impliquées dans la réaction inflammatoire (TNF- $\alpha$, interleukinc 1 $($ IL-1)) [2, 3] et autres médiateurs $\left(\mathrm{LTB}_{4}\right.$, platelet-activating factor ou PAF, etc.).

\section{Syndrome de détresse respiratoire de l'adulte}

Il s'agit d'un syndrome où un événement "catastrophique " de nature variée (Tableau 1) [4] sera suivi du développcment d'unc atteinte alvćolaire diffusc, caractérisée par une altération de la perméabilité, un odème $\mathrm{m} / \mathrm{s} n^{\circ} 10$, vol. 7, décembre 91

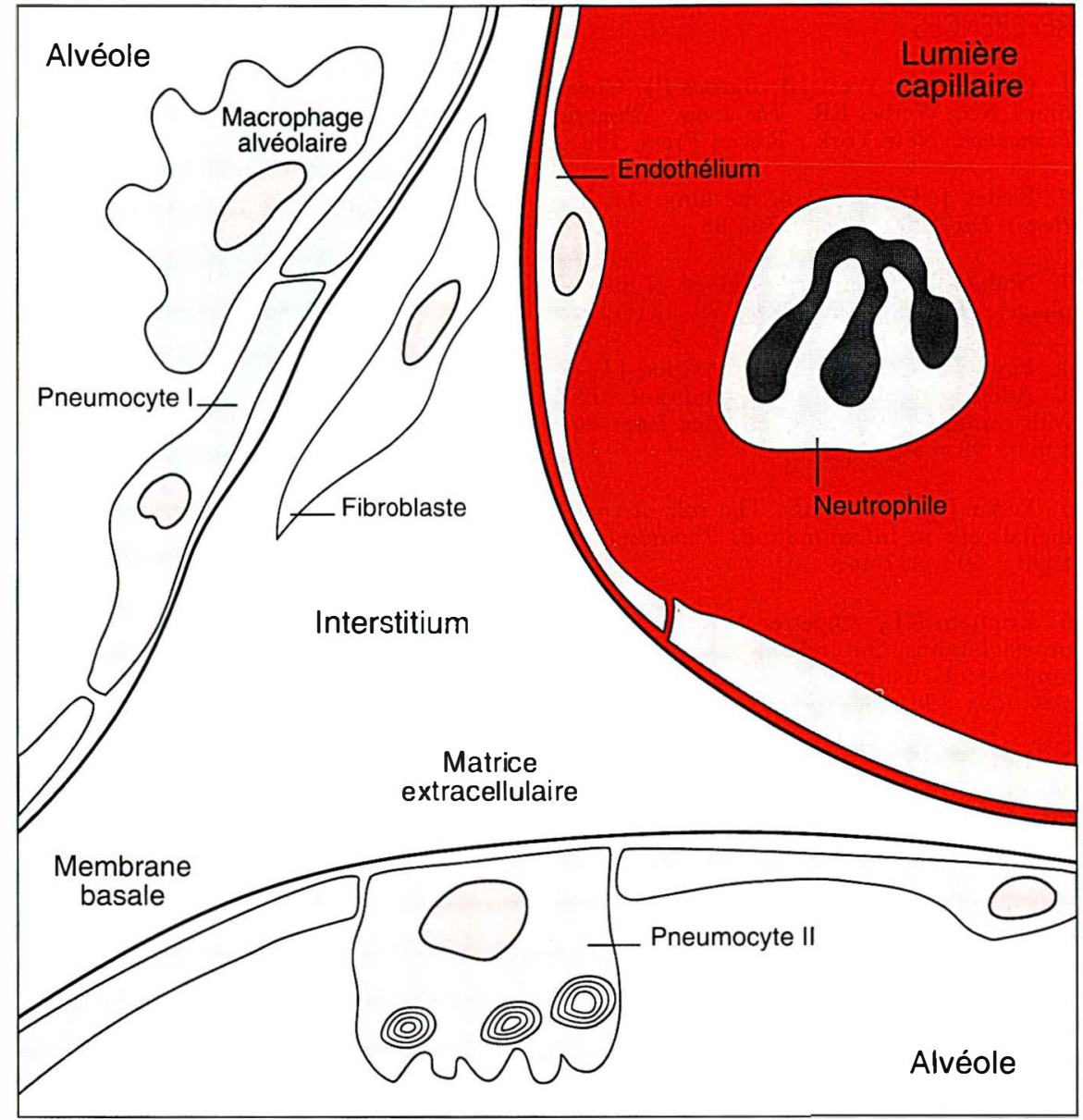

Figure 1. Représentation schématique des espaces alvéolo-capillaires et de leurs constituants cellulaires principaux. Entre les alvéoles et la lumière capillaire se trouve l'interstice, composé de matrice extracellulaire et de fibroblastes.

Tableau I

CONDITIONS ASSOCIÉES A LA SURVENUE DU SYNDROME DE DÉTRESSE RESPIRATOIRE DE L'ADULTE (d'après Fowler et al. [4])

\begin{tabular}{lr} 
Circulation extracorporelle & \\
Brûlure & $1,7 \%$ \\
Bactériémie & $2,3 \%$ \\
Transfusions multiples & $3,8 \%$ \\
Fractures & $4,6 \%$ \\
Pneumonies & $5,3 \%$ \\
Coagulopathie intravasculaire disséminée & $11,9 \%$ \\
Aspiration pulmonaire & $22,2 \%$ \\
\hline
\end{tabular}

interstitiel et alvéolaire, des lésions endothéliales et épithéliales, et l'organisation de l'cxsudat par des fibroblastes qui prolifèrent et sécrètent du collagènc. Cet état est potentiellement réversible, mais, malheurcuscment, au décours de cette affection, la plupart des paticnts vont développer une défaillance multi-viscćralc (multiple organ failure), de caractère souvent fatal. Si le tableau clinique répond à une définition bien codifiée, sa patho- 


\section{RÉFÉRENCES}

1. Crystal RG, West JB, Barnes PJ, Cherniack NS, Weibel ER. The Lung: Scientific Foundation. New York: Raven Press, 1991.

2. Kelley J. Cytokines of the lung. Am Rev Respir Dis 1990 ; 141 : 765-88.

3. Nathan CF. Secretory products of macrophages. J Clin Invest 1987 ; 79 : 319-26.

4. Fowler AA, Hamman RF, Good JT, et al. Adult respiratory distress syndrome : risk with common predispositions. Ann Intem Med $1983 ; 98: 593-7$.

5. Pober JS, Cotran RS. The role of endothelial cells in inflammation. Transplantation 1990 ; 50 : 537-44

6. Brigham KL, Ogletree ML. Effects of prostaglandins and related compounds on lung vascular permeability. Bull Europ Physiopath Resp $1981 ; 17$ : 703-22.

7. Lewis MS, Whatley RE, Cain P, McIntyre TM, Prescott SM, Zimmerman GA. Hydrogen peroxide stimulates the synthesis of platelet-activating factor by endothelium and induces endothelial celldependent neutrophil adhesion. J Clin Invest $1988 ; 82: 2045-55$.

8. Patel KD, Zimmerman GA, Prescott SM, McEver RP, McIntyre TM. Oxygen radicals induce human endothelial cells to express GMP-140 and bind neutrophils. J Cell Biol 1991; 112 : 749-59.

9. Strieter RM, Kunkel SL, Showell HJ, et al. Endothelial cell gene expression of a neutrophil chemotactic factor by TNF-alpha, LPS, and IL-1beta. Science 1989; 243 : 1467-9.

10. Brieland JK, Kunkel RG, Fantone JC Pulmonary alveolar macrophage function during acute inflammatory lung injury. $\mathrm{Am}$ Rev Respir Dis 1987; 135 : 1300-6.

11. Guthrie LA, McPhail LC, Henson PM, Johnston RB Jr. Priming of neutrophils for enhanced release of oxygen metabolites by bacterial lipopolysaccharide. Evidence for increased activity of the superoxideproducing enzyme. J Exp Med $1984 ; 160$. 1656-71.

12. Fowler AA, Hyers TM, Fisher BJ, Bechard DE, Centor RM, Webster RO. The adult respiratory distress syndrome. Cell populations and soluble mediators in the air spaces of patients at high risk. Am Rev Respir Dis 1987 ; 136 : 1225-31.

13. Ognibene FP, Martin SE, Parker MM, et al. Adult respiratory distress syndrome in patients with severe neutropenia. $N$ Engl $J$ Med 1986 ; 315 : 547-51.

14. Malik AB, Horgan MJ. Mechanisms of thrombin-induced lung vascular injury and edema. Am Rev Respir Dis 1987; 136 467-70. génie précise reste peu claire. Il y a, pourtant, de nombreux modèles expérimentaux qui sont à même de reproduire les lésions initiales de type "lésion microvasculaire " ou "lésion alvéolaire diffuse ", mais qui ne rendent pas compte du décours de ce syndrome sur plusieurs jours voire plusieurs semaines. La multiplicité de ces modèles expérimentaux reflète le caractère multifactoriel de cette affection. La complexité et la diversité des éléments pathogéniques expliquent, par ailleurs, l'insuccès actuel des programmes thérapeutiques à visée causale, fondés sur l'utilisation d'un agent, qu'il s'agisse des corticoïdes ou de prostaglandines.

Dans cette esquisse de synthèse, la plupart des données rapportées ont été obtenues lors d'expériences aiguës portant sur des animaux ou dans des

protocoles utilisant des populations cellulaires isolées ou en culture. Leurs relations avec la détresse respiratoire de l'adulte, si elles ont le mérite de la cohérence ou de la vraisemblance sur le plan biologique, gardent cependant un caractère parfois spéculatif, en raison de l'absence de réels modèles expérimentaux ainsi que cela vient d'être mentionné.

\section{Phase d'initiation (figure 2)}

Sous l'effet de stimuli ou d'agressions variés, dont l'endotoxine bactérienne ou lipopolysaccharide qui jouerait un rôle majeur, mais non exclusif, deux phénomènes se produisent.

(1). La réponse endothéliale [5], qui comprend : (a) la libération de prostanoïdes, dont le thromboxane $\mathrm{A}_{2}$ $\left(\mathrm{TxA}_{2}\right)$, responsable d'une réaction

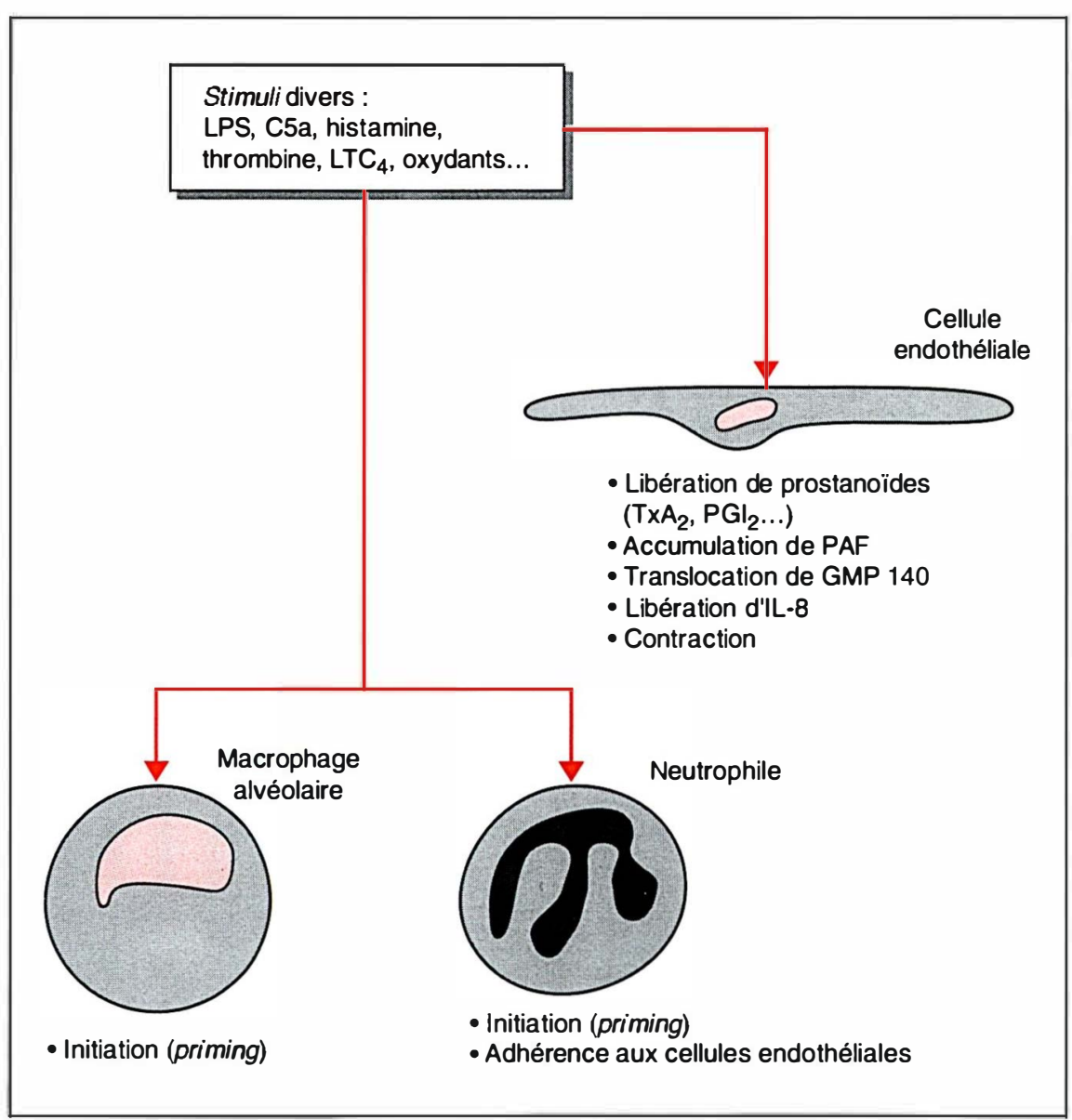

Figure 2. Diagramme illustrant la phase initiale de l'atteinte endothéliale et de l'" initiation " de cellules inflammatoires lors d'une atteinte pulmonaire aiguë sous l'effet de divers stimuli. 
vaso-constrictrice pulmonaire précoce [6] et la prostacycline ou $\mathrm{PGI}_{2}$, l'accumulation de PAF, la translocation de la GMP 140 (granular membrane protein capable de jouer le rôle de "ligand " pour l'adhérence des neutrophiles) $[7,8]$; (b) la libération d'IL-8, une cytokine dotée de propriétés chémo-attractives pour les mêmes neutrophiles [9].

(2). L' initiation" (ou priming) des cellules inflammatoires (macrophages alvéolaires et neutrophiles), qui modifient leur état métabolique de telle sorte que leur sensibilité à un stimulus secondaire est considérablement accrue [10, 11]. L'implication des neutrophiles, même si elle est considérée comme la règle [12], n'est pas une condition absolument nécessaire. Certains cas de détresse respiratoire de l'adulte ont été rapportés chez des patients neutropéniques [13], ce qui illustre le fait que des mécanismes autres que l'activation des neutrophiles peuvent opérer dans la genèse de ce syndrome (activation du complément, coagulation intravasculaire par exemple) [14].

L'expression fonctionnelle sera, ainsi qu'il a déjà été mentionné, une réaction hypertensive pulmonaire due principalement à la libération de $\mathrm{TxA}_{2}$, réaction que l'on peut bloquer par des inhibiteurs de la cyclooxygénase, telle l'indométhacine. L'atteinte endothéliale se marquera par la survenue d'un œdème avant tout interstitiel. Il en résultera des troubles d'échanges gazeux et une diminution de la compliance pulmonaire. Ce stade est réversible.

\section{Phase aiguë inflammatoire (figure 3)}

Dans cette deuxième phase, de caractère également aigu, mais dont l'expression, liée à un stimulus secondaire ou prolongé, requiert quelques heures, l'événement majeur devient l'activation du macrophage alvéolaire préalablement "initié ", avec la sécrétion de cytokines (TNF- $\alpha$, IL-1) qui conditionneront toute une série de réactions :

- stimulation d'IL-8 par différentes populations cellulaires et renforcement de la chémo-attraction pour les neutrophiles [15] ;

- induction et expression de plusieurs $\mathrm{m} / \mathrm{s} n^{\circ} 10$, vol. 7, décembre 91 molécules d'adhérence, dont l'ELAM (endothelial leukocyte adhesion molecule), une glycoprotéine de $110 \mathrm{kDa}$ appartenant au groupe des sélectines, ainsi qu'une autre glycoprotéine, membre de la superfamille des immunoglobulines, l'ICAM-1 (intercellular adhesion molecule), servant de "contrerécepteur " à la molécule d'adhérence leucocytaire LFA-1 $[5,16,17]$.

Sous l'effet concomitant du leucotriène LTB4 et du PAF que les macrophages peuvent aussi libérer, les neutrophiles vont donc pouvoir adhérer aux cellules endothéliales et, par l'entremise des protéases et dérivés toxiques de l'oxygène qu'ils libèrent lors de leur activation, les léser. Cette atteinte cellulaire se retrouve aussi au niveau des cellules épithéliales alvéolaires avec possibilité de dénudation de la membrane basale. L'atteinte endothéliale et l'activation plaquettaire qui y est associée sont également incriminées dans la formation de microthrombi que l'on trouve fréquemment à ce stade précoce de la maladie, comme dans sa phase plus tardive.

Les conséquences physiologiques de cette deuxième phase sont essentiellement les mêmes que celles précédemment citées, tout en ayant un caractère de gravité plus marqué en rapport avec le développement de l'œdème interstitiel en œdème alvéolaire du fait de l'atteinte endothéliale et épithéliale.

\section{Phase de réparation}

En même temps que se succèdent ou se poursuivent ces agressions qui font que l'ARDS (adult respiratory detress syndrome) représente plutôt un conti-

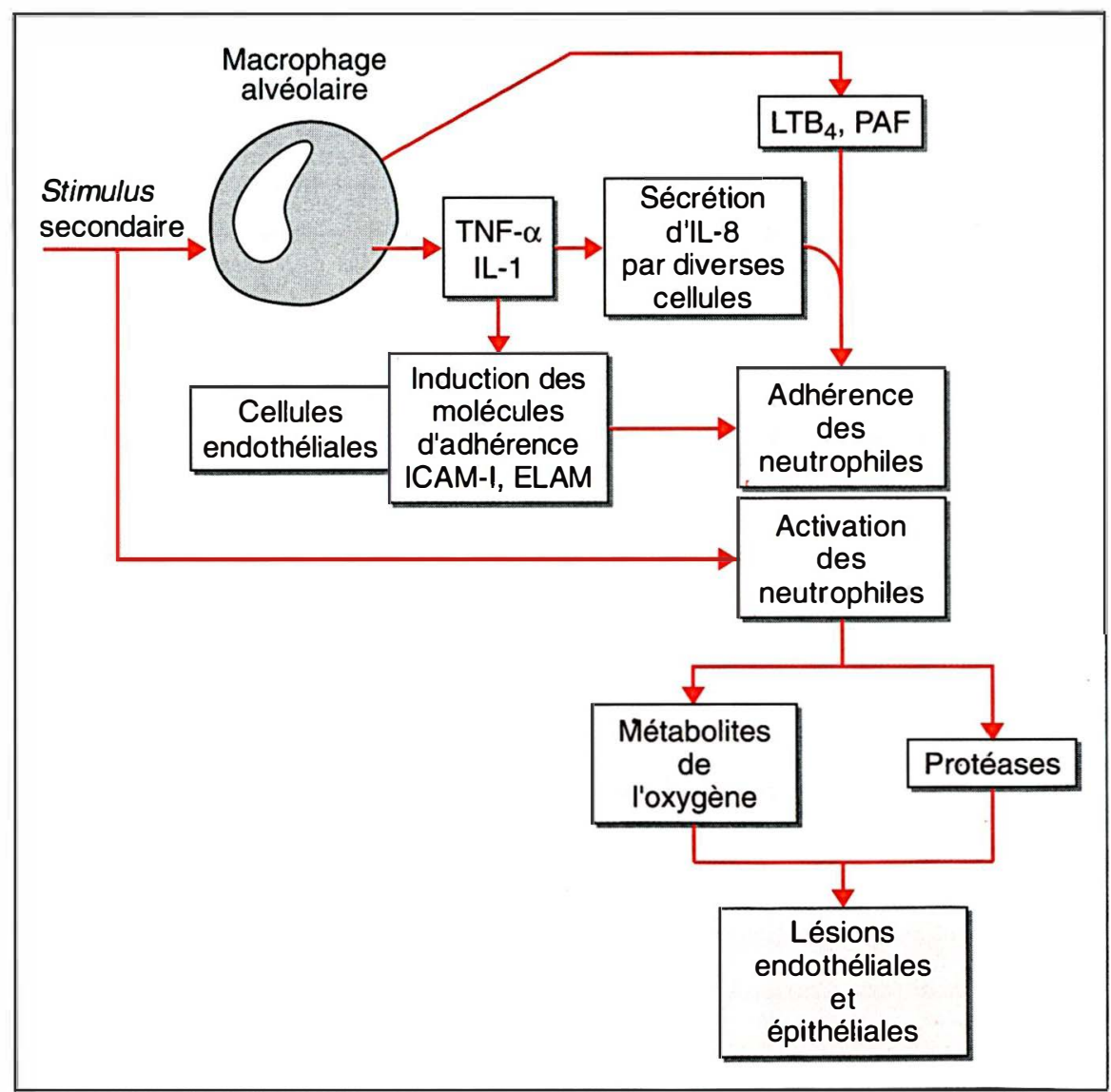

Figure 3. Représentation schématique de la séquence des événements suivant un stimulus secondaire ou continu et menant à l'activation des macrophages alvéolaires, l'induction de molécules d'adhérence, la chémoattraction et l'activation des neutrophiles, le développement de lésions endothéliales et épithéliales. 


\section{RÉFÉRENCES}

15. Standiford TJ, Kunkel SL, Basha MA, et al. Interleukin-8 gene expression by a pulmonary epithelial cell line. A model for cytokine networks in the lung. $J$ Clin Invest $1990 ; 86: 1945-53$

16. Bevilacqua MP, Stengelin S, Gimbrone MA Jr, Seed B. Endothelial-leukocyte adhesion molecule 1 : an inducible receptor for neutrophils related to complement regulatory proteins and lectins. Science 1989 243 : 1160-5.

17. Carlos TM, Harlan JM. Membrane proteins involved in phagocyte adherence to endothelium. Immunol Rev 1990 ; 114 : 5-28.

18. Sporn MB, Roberts AB, Wakefield LM, DeCrombrugghe B. Some recent advances in the chemistry and biology of transforming growth factor-beta. J Cell Biol 1987 ; 105 : 1039-45.

19. Khalil N, Bereznay O, Sporn M, Greenberg AH. Macrophage production of transforming growth factor beta and fibroblast collagen synthesis in chronic pulmonary inflammation. $J$ Exp Med 1989 ; 170 : 727-37.

20. Bitterman PB, Wewers MD, Rennard SI, Adelberg S, Crystal RG. Modulation of alveolar macrophage-driven fibroblast proliferation by alternative macrophage mediators. J Clin Invest $1986 ; 77: 700-8$.

21. Nagaoka I, Trapnell BC, Crystal RG. Upregulation of platelct-derived growth factor-A and - $\mathrm{B}$ gene expression in alveolar macrophages of individuals with idiopathic pulmonary fibrosis. J Clin Invest $1990 ; 85$ : 2023-7.

22. Libby $P$, Friedman GB, Salomon RN. Cytokines as modulators of cell proliferation in fibrotic diseases. Am Rev Respir Dis 1989 ; $140: 1114-7$.

23. Antoniades HN, Bravo MA, Avila RE, et al. Platelet-derived growth factor in idiopathic pulmonary fibrosis. $J$ Clin Invest $1990 ; 86$ : 1055-64.

24. Akahoshi T, Oppenheim JJ, Matsushima K. Interleukin-1 stimulates its own receptor expression on human fibroblasts through the endogenous production of prostaglandin(s). J Clin Invest $1988 ; 82$ : 1219-24.

25. Katzenstein A-LA, Myers JL, Mazur MT. Acute interstitial pneumonia. A clinicopathologic, ultrastructural, and cell kinetic study. Am J Surg Pathol 1986; 10 : 256-67.

26. Kuhn III C, Boldt J, King TE Jr, Crouch E, Vartio T, McDonald JA. An immunohistochemical study of architectural remodeling and connective tissue synthesis in pulmonary fibrosis. Am Rev Respir Dis $1989 ; 140$ : 1693-703. nuum qu'une série bien délimitée de phases, se développe progressivement un deuxième type de réaction, caractérisé par des processus de réparation au niveau épithélial (hyperplasie des pneumocytes II) et le remodelage de l'interstitium, des espaces alvéolaires et des vaisseaux par une prolifération de cellules mésenchymateuses.

\section{Fibrose alvéolaire (figure 4)}

Les facteurs pathogéniques impliqués dans la prolifération fibroblastique et l'accumulation de collagène au cours de cette phase subaiguë de réparation sont probablement multiples. Leur identification n'est, le plus souvent, pas directe, mais provient de données expérimentales acquises dans l'étude des fibroses idiopathiques humaines et de modèles expérimentaux [18-22].

Parmi les substances impliquées, citons :

1. celles qui ont une action favorisant la migration et diapédèse des fibroblastes, dont, en particulier : le transforming growth factor $\beta$ (TGF- $\beta$ ), la fibronectine, le platelet-derived growth factor (PDGF) ;

2. celles qui stimulent la division des cellules mésenchymateuses, dont : la fibronectine, l'interleukine 1, le TNF$\alpha$, le PDGF, l'insulin-growth factor-1 (IGF-1).

L'origine de ces cytokines est multiple. $\mathrm{Si}$ le macrophage alvéolaire apparaît jouer un rôle pivot, ce dernier n'est pas exclusif, et les cellules épithéliales et endothéliales [23], ainsi que les plaquettes et les fibroblastes eux-mêmes, peuvent contribuer, en partie tout au moins, à la modulation de ce processus et au "réseau des cytokines" (cytokine network). Il est également important de garder à l'esprit le fait que l'IL-1 joue un rôle autocrine en ce sens que cette monokine, une fois libérée, peut stimuler l'expression de ses récepteurs au niveau de la cellule même qui la synthétise [24].

Des études réalisées avec la thymidine marquée indiquent que la prolifération cellulaire a souvent un caractère focal [25]. Il en est de même pour la synthèse de constituants de la matrice extracellulaire.

La formation de tissu fibrotique se fait selon un nombre limité de stéréotypes, correspondant essentiellement à trois modèles qui peuvent être asso- ciés [26] : (1) prolifération fibroblastique à l'intérieur des septa alvéolaires ; (2) colonisation et organisation de l'exsudat alvéolaire par ces mêmes fibroblastes ; (3) fibrose sur induration atélectatique, où les parois alvéolaires dénudées s'accolent et forment la base d'une réaction fibreuse où la structure alvéolaire est perdue [27]. 3. On peut enfin citer les substances qui stimulent la formation des composantes de la matrice extracellulaire dont la fibronectine, le collagène, et inhibent la synthèse des protéases capables de les dégrader. C'est le TGF- $\beta$ qui paraît jouer le rôle le plus important dans ce phénomène qui, dans le syndrome de détresse respiratoire de l'adulte, n"a pas l'importance qu'on lui reconnaît dans l'affection plus lentement progressive que représente la fibrose idiopathique.

Sur le plan physiopathologique, cette prolifération fibroblastique (sans oublier celle des myofibroblastes et des cellules musculaires lisses) et la formation de fibres collagènes vont modifier les propriétés élastiques du poumon et participer à l'augmentation de la pression de rappel du tissu pulmonaire, expression de la rigidité ainsi acquise du parenchyme.

\section{Remodelage vasculaire}

Certains cas de syndrome de détresse respiratoire de l'adulte, dont le nombre croît avec la durée de la maladie, sont caractérisés par une augmentation nette de la pression artérielle pulmonaire en rapport avec une diminution marquée du nombre des vaisseaux pulmonaires [28]. A côté des phénomènes de microthrombose précédemment cités, une des particularités de ce stade, en général assez tardif, consiste en un remodelage de la paroi vasculaire avec une augmentation des cellules mésenchymateuses dans les parois vasculaires, qu'il s'agisse de fibroblastes, de myofibroblastes, de cellules musculaires lisses ou de péricytes. La conséquence en est une diminution de la surface luminale et une muscularisation des petits vaisseaux qui, toutes deux, contribuent à l'établissement de ce régime d'hypertension.

La pathogénie de ces changements est en général attribuée aux plaquettes activées au contact d'un endothélium 


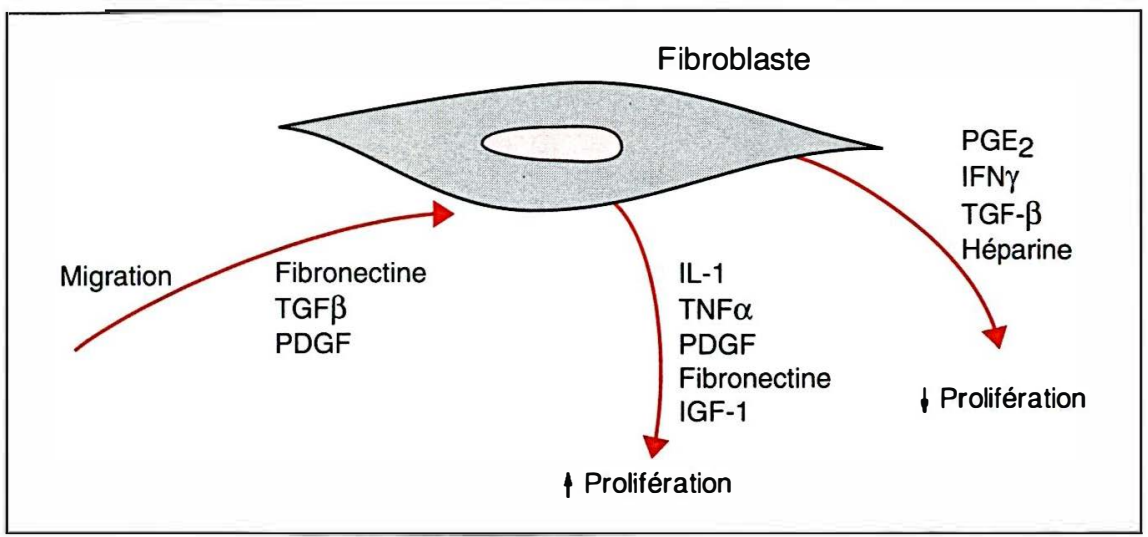

Figure 4. Représentation schématique de différents facteurs impliqués dans la migration et la prolifération des fibroblastes au niveau pulmonaire.

lésé et à leur production de PDGF et de TGF- $\beta$. Les cellules endothéliales peuvent être aussi induites à produire de telles cytokines et à participer ainsi à ce remodelage [29].

Les conséquences physiologiques en sont dramatiques. A côté du stress que représente l'hypertension pulmonaire pour le cœur droit, la diminution du lit vasculaire disponible augmente l'espace mort ( = ventilé, mais non perfusé). Dans une condition où les volumes pulmonaires et donc la ventilation-minute sont sérieusement limités par la fibrose parenchymateuse, cette augmentation de l'espace mort physiologique va se faire au détriment de la ventilation alvéolaire et amener à une rétention de $\mathrm{CO}_{2}$, qui s'ajoute à l'hypoxie.

\section{Autres éléments pathogéniques possibles}

Cette rapide revue, nécessairement incomplète, n'a pu inclure tous les éléments impliqués dans la pathogénie de cette entité. Il n'a pas été fait mention de l'IL-6 qui, sous l'action d'autres monokines (IL-1, TNF- $\alpha$ ), peut être produite par une multitude de types cellulaires et amplifier ainsi la réaction inflammatoire [30]. Le rôle des lymphocytes n'a pas été mentionné, alors même qu'il est vraisemblable que, du fait de l'activation des macrophages alvéolaires, ils soient également stimulés. Le problème des rôles respectifs des protéases libérées par les neutrophiles et les macropha$\mathrm{m} / \mathrm{s} n^{\circ}, 10$, vol. 7 , décembre 91 cas pourtant, et dans certaines conditions seulement, pour le TGF- $\beta$, ainsi que pour l'interféron $\gamma$ et l'héparine. Plus intrigante est l'action paradoxale inhibitrice sur la prolifération des fibroblastes résultant de l'administration combinée de l'IL-1 et du TNF- $\alpha$, alors que, prise isolément, chacune de ces cytokines a un pouvoir mitogénique positif [33]. C'est apparemment par le biais d'une sécrétion augmentée des $\mathrm{PGE}_{2}$ que s'explique ce phénomène, les $\mathrm{PGE}_{2}$ ayant un effet inhibiteur bien connu sur la réplication des fibroblastes. Elles ont également la propriété de diminuer l'expression de l'ARN messager du TNF- $\alpha$ au niveau des macrophages, à l'instar du TGF$\beta[34,35]$. L'IL-4 a également le potentiel de réduire la synthèse et du TNF- $\alpha$ et de l'IL-1 par ces mêmes macrophages [36]. Force est, cependant, de constater la pauvreté apparente de la boucle du rétrocontrôle négatif dans la régulation de l'inflammation et de la fibrogenèse.

Dans une autre perspective, il paraît important de souligner qu'il y a d'autres façons de diminuer l'impact des cytokines responsables du caractère inflammatoire de cette affection et dotées d'effets mitogènes pour les fibroblastes. L'existence d'antagonistes de l'IL-I et du TNF- $\alpha$ vient d'être démontrée [37], qu'il s'agisse d'une protéine spécifique agissant au niveau du récepteur, comme cela semble être le cas pour l'IL-1 [38], ou que l'antagoniste soit lui-même un constituant soluble du récepteur, ainsi que cela apparaît être le cas pour le TNF- $\alpha[39]$. Si la présence de ces inhibiteurs ou antagonistes a été démontrée dans le milieu conditionné par des macrophages alvéolaires humains prélevés lors d'un lavage broncho-alvéolaire, leur rôle dans la terminaison d'une réaction inflammatoire n'est pas encore fermement établi. L'étude de la cinétique de leur production dans des situations pathologiques bien définies permettra sans doute de mieux comprendre leur implication potentielle dans la modulation de l'inflammation.

\section{Conclusions}

Le syndrome de détresse respiratoire de l'adulte représente un modèle par- 


\section{RÉFÉRENCES}

27. Burkhardt A. Alveolitis and collapse in the pathogenesis of pulmonary fibrosis. $A m$ Rev Respir Dis 1989 ; 140 : 513-24.

28. Zapol WM, Jones R. Vascular components of ARDS. Clinical pulmonary hemodynamics and morphology. Am Rev Respir Dis 1987 ; 136 : 471-4.

29. Roberts AB, Sporn MB. Regulation of endothelial cell growth, architecture, and matrix synthesis by TFG-beta 1. Am Rev Respir Dis 1989 ; 140 : 1126-8.

30. Kotloff RM, Little J, Elias JA. Human alveolar macrophage and blood monocyte interleukin-6 production. Am J Respir Cell Mol Biol 1990 ; 3 : 497-505.

31. Jornot L, Mirault ME, Junod AF. Protein synthesis in hyperoxic endothelial cells : evidence for translational defect. $J A p p$ Physiol 1987 ; 63 : 457-64.

32. Jornot L, Junod AF. Hypoxanthinexanthine oxidase-related defect in polypeptide chain initiation by endothelium. $J A p p l$ Physiol 1989 ; 66 : 450-7.

33. Elias JA Gustilo K Freundlich B Human alveolar macrophage and blood monocyte inhibition of fibroblast proliferation. Evidence for synergy between interleukin-1 and tumor necrosis factor. $A m$ Rev Respir Dis 1988 ; 138 : 1595-603.

34. Scales WE, Chensue SW, Otterness I, Kunkel SL. Regulation of monokine gene cxpression: prostaglandin $\mathrm{E}_{2}$ suppresses tumor necrosis factor but no interleukin-1alpha or beta-mRNA and cellassociated bioactivity. J Leukoc Biol 1989 45 : 416-21.

35. Espevik T, Figari IS, Shalaby MR, et al. Inhibition of cytokine production by cyclosporin A and transforming growth factor beta. J Exp Med 1987; 166 : 571-6.

36. Essner R, Rhoades K, McBride WH Morton DL, Economou JS. IL-4 downregulates IL-1 and TNF gene expression in human monocytes. J Immunol $1989 ; 142$ : 3857-61.

37. De Rochemonteix-Galve B, Dayer J-M, Junod AF. Fibroblast-alveolar cell interactions in sarcoidosis and idiopathic pulmonary fibrosis : evidence for stimulatory and inhibitory cytokine production by alveolar cells. Eur Respir J 1990; 3 : 653-64.

38. Galve-de Rochemonteix B, Nicod LP, Junod AF, Dayer J-M. Characterization of a specific 20 - to $25-\mathrm{kD}$ interleukin-1 inhibitor from cultured human lung macrophages. Am J Respir Cell Mol Biol 1990 ; 3 : 355-61.

39. Seckinger $\mathrm{P}$, Zhang J-H, Hauptman B Dayer J-M. Characterization of a TNFalpha inhibitor. Evidence for immunological crossreactivity with the TNF receptor. Proc Natl

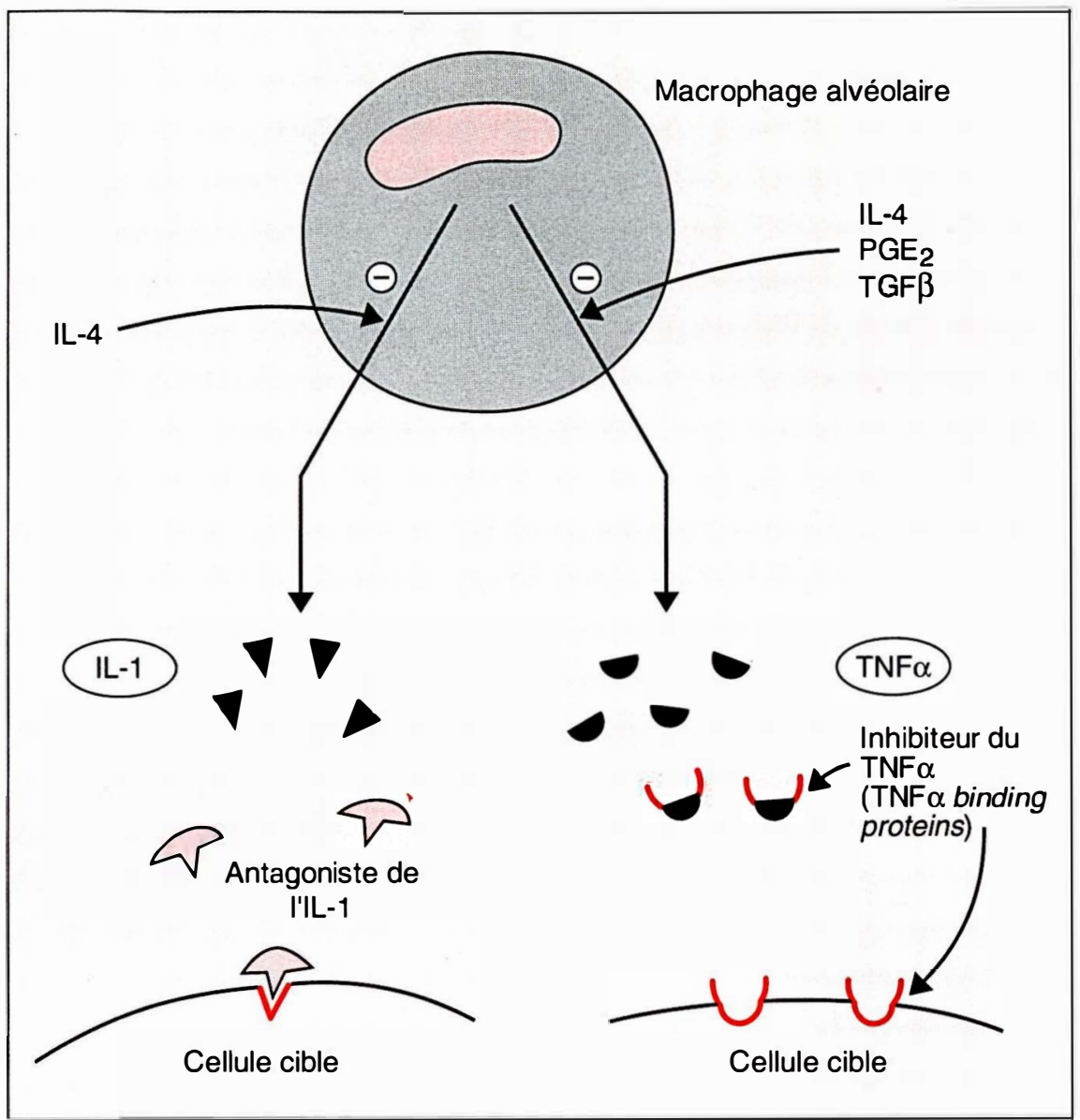

Figure 5. Diagramme illustrant le mécanisme potentiel impliqué dans la résolution de l'inflammation. Les deux points d'impact principaux sont la diminution de l'expression des cytokines au niveau du macrophage alvéolaire et la production d'antagonistes ou inhibiteurs de ces mêmes cytokines.

ticulièrement intriguant de la pathologie humaine, qui n'a, pour le moment, pas d'équivalent animal ou expérimental, d'où les difficultés inhérentes à son étude et à l'analyse des facteurs impliqués. Il se dégage cependant un certain nombre d'éléments qui semblent jouer un rôle majeur dans l'établissement de ce syndrome et qu'il paraît peut-être utile de résumer brièvement :

- une agression continue ou répétitive (endotoxine par exemple) ;

- l'initiation, puis l'activation des cellules inflammatoires (macrophages alvéolaires, neutrophiles) ;

- la participation des cellules endothéliales à l'adhérence des neutrophiles dans les microvaisseaux et à la réaction inflammatoire :

- une cascade de sécrétion de cytoki- nes qui permettent une amplification de la réaction inflammatoire et son auto-entretien par plusieurs populations cellulaires ;

- l'atteinte de la perméabilité et le remodelage des espaces alvéolocapillaires qui sont les données pathologiques majeures;

- la possibilité de résolution complète, par des mécanismes encore mal élucidés (rôle des $\mathrm{PGE}_{2}$, de l'IL-4 et des antagonistes de l'IL-1 et du TNF- $\alpha$ ?).

Une meilleure compréhension de ces facteurs pathogéniques est nécessaire si l'on veut aborder le problème de la prévention et du traitement de ce syndrome autrement que par un empirisme aléatoire et des mesures de soutien héroïques, mais d'une efficacité relative 


\section{Summary}

Biology and physiology of the adult respiratory distress syndrome

The results of recent studies have shed light on some of the mechanisms involved in the pathogenesis of the adult respiratory distress syndrome (ARDS). Following exposure to various stimuli (especially endotoxin), endothelial cells undergo changes enabling them to attract neutrophils and promote their adhesion, at the same time as inflammatory cells are primed. Upon secondary or continuous stimulation, alveolar macrophages release cytokines such as IL-1 and TNF- $\alpha$ which in turn stimulate other cell types to produce other cytokines or to induce adhesion molecules. Neutrophils, once adherent and activated, can damage endothelial cells and epithelial cells through the release of $\mathrm{O}_{2}$ metabolites and proteases. The third phase is that of repair, with the migration and proliferation of epithelial cells and mesenchymal cells under the action of various cytokines, resulting in the remodelling of both the alveolar structures and the vessels. Resolution can finally take place, its mechanisms are unclear, although $\mathrm{PGE}_{2}, \quad \mathrm{IL}-4$ and IL- 1 and TNF- $\alpha$ inhibitors are good candidates to terminate the inflammatory reaction.

TIRÉS A PART

A.F. Junod.

$\mathrm{m} / \mathrm{s} n^{\circ}$ 10. vol. 7, décembre 91 